\title{
TERDURANTISM: A VIABLE THEORY OF PERSISTENCE?
}

\author{
Francis Kenneth P. Raterta \\ De La Salle University, Philippines
}

\begin{abstract}
Theories of persistence are often motivated on the grounds that they can account for or solve certain problems that accompany persistence as a metaphysical problem. These problems are what I will call the problems of persistence: change, cohabitation, and vagueness. In this paper, I claim that any theory of persistence should be able to account for these problems. Any theory of persistence which fails to do so should be rejected or, at the very least, be seen as unsatisfactory. Kristie Miller introduces a possible contender, terdurantism, which is a "non-perdurantist fourdimensionalism," as she puts it. This view is attractive because it avoids the usual objections raised against its rivals: perdurantism and endurantism. Miller and I both ultimately argue against the plausibility of terdurantism as a theory of persistence, but our motivations differ. Miller's argument is based on the presumption that any theory of persistence which is non-perdurantist ultimately fails. She argues that temporal parts are needed in any account of persistence. My argument is based on the "problem-solving" capacity or the ability of the theory to account for the persistence problems. I will argue that terdurantism is not a plausible theory of persistence since it fails to give a viable account of the three problems. By showing the importance of accounting for the three problems - and how a terdurantist position fails to accomplish this - I hope to have convinced the reader that not only is terdurantism an unattractive persistence theory, but any plausible theory of persistence ought to account for the problems.
\end{abstract}

\section{INTRODUCTION}

A prevalent metaphysical issue has to do with an object's existence at various times. This issue is called persistence, and the main contention is on how objects exist at different times. More specifically, in virtue of what do objects persist? Traditionally, there are two main theories which explain how objects persist: endurantism (the view that objects lack temporal parts and persist by being wholly present at each time it exists) and perdurantism (the view that objects are extended in time and persist by having distinct temporal parts). Both theories are often motivated on the grounds that 
they can account for specific problems that persistence gives rise to. These problems are change, cohabitation, and vagueness.

In considering the three problems of persistence, I claim that any theory of persistence should give a tenable account of the aforementioned problems. This move is crucial as it will serve as my main premise in arguing that terdurantism, or "nonperdurantist four-dimensionalism," as Kristie Miller $(2009,624)$ calls it, is not a plausible view of persistence. In this paper, I argue that terdurantism is not a viable persistence theory because it fails to account for specific problems brought about by persisting objects. By assessing and comparing how it accounts for these problems, we see how it falls short of its rivals when it comes to explanatory power. But more importantly, and the chief objective of this paper, in showing that terdurantism fails to accomplish what its rivals can, any persistence theory ought to account for these problems lest they be rejected or seen as unsatisfactory.

In Section I, I discuss the main theories of persistence: endurantism, and perdurantism as well as clarify some relevant concepts that help the reader avoid any unnecessary confusion regarding terminology. In Section II, I discuss the three problems of persistence briefly and explain why giving an account of these problems is important as far as theories of persistence are concerned. Perdurantist and endurantist accounts of the problem will also be discussed here. In section III, I discuss terdurantism as well as Miller's motivations for it. In section IV, I present my main argument and motivate its premises. Possible objections, as well as my replies, are presented in section V. How terdurantism might account for the three problems, as well as objections to each are discussed in section VI.

\section{PERSISTENCE}

As touched upon earlier, persistence has to do with an object's existence at different times. But what exactly does it mean to say that an object persists? In other words, what does it mean for an object to exist at different times? David Lewis (1986, 202) says an object persists if and only if "it exists at various times." This is not controversial as far as our theories of persistence are concerned since both would agree that when an object persists, "something" exists at different times. The question now is what this "something" is. For the perdurantist, what exists at different times are temporal parts of an object. On the other hand, the endurantist would say that what exists at different times is the object itself, as a whole (wholly present), and not merely its parts. Let us explore these ideas further.

\section{Four-Dimensionalism}

Perdurantism is a theory of persistence that argues that objects are extended not just in space but also in the fourth dimension, time. Because of this, some philosophers use perdurantism and four-dimensionalism interchangeably (the same applies to endurantism and three-dimensionalism) or argue that four-dimensionalism entails perdurantism. However, there are philosophers, such as Josh Parsons, who question this entailment. Parsons $(2000,404)$ says that four-dimensionalism and three- 
dimensionalism are theories of extension, whereas perdurantism and endurantism are theories of persistence. Furthermore, he $(2000,415)$ argues that four-dimensionalism does not entail perdurantism and that it can be compatible with endurantism as well. Although the ideas of Parsons will not play a role in this paper, I mentioned them now to say that I maintain the entailment that holds between theories of extension and theories of persistence.

Theodore Sider $(2008,242-243)$ lists down three ways in which time is analogous to space, but I would like to focus specifically on the parthood analogy: the analogy between spatial and temporal parts. Sider says, "temporal parts theory is the claim that time is like space in one particular respect, namely, with respect to parts." To illustrate this point, let us have an example. Consider my dog, Max, a spatially extended entity. He has different spatial parts such as his head, limbs, torso, and tail. Notice that these parts are smaller in size compared to the whole object. According to the temporal parts theory, Max is also temporally extended and has corresponding temporal parts. Again, these temporal parts are much smaller or "shorter-lived" than the whole temporal object. A temporal part of Max could be his year-2015 temporal part. This part is smaller than the whole persisting object, Max. This temporal part is exactly the same as the spatial entity which exists at that time, Max, the puppy, but it only exists at that moment. As Sider $(2008,242)$ says, "temporal parts have spatial parts, and spatial parts have temporal parts." Given this relationship, Sider $(1997,8)$ defines an instantaneous temporal part, $\mathrm{x}$ is defined as:

$\mathrm{x}$ is an instantaneous temporal part of $\mathrm{y}$ at instant $\mathrm{t}=\mathrm{df}$. i) $\mathrm{x}$ exists at, but only at $t$, ii) $x$ is a part of $y$ at $t$, and iii) $x$ overlaps everything that is part of $y$ at $t$.

Applying this definition to my example of Max, the 2015 temporal part of Max exists only at that time, is a part of Max at that time, and overlaps everything that is part of Max at that time (e.g., his tail, legs, torso, etc.). This definition of a temporal part ensures that the temporal part is as big as the object itself at that time. An object then persists by perduring à la Lewis $(1986,202)$ iff "it persists by having different [instantaneous] temporal parts. . .at different times, though no one part of it is wholly present at more than one time."

\section{Three-Dimensionalism}

Before we discuss the central claim of three-dimensionalism regarding persisting objects, it is important to note that it is possible for us to have a threedimensional picture that is consistent with objects being extended in space as well as time. For instance, we can consider a spacetime region occupied by flatlanders wherein there are only two spatial dimensions and one temporal dimension. The flatlander is extended in time but is still extended in only three dimensions. However, a consistent three-dimensionalist would say that the flatlander is not temporally extended. Rather, they only bear certain properties in relation to times. Let us say that Max is a flatland dog, and he is hungry at $\mathrm{t} 1$ and full at $\mathrm{t} 2$. The three-dimensionalist 
would say that Max only bears certain time-indexed properties (i.e., hungry-at-t1 and full-at-t2) but he is not temporally extended. Objects are only extended in the three spatial dimensions.

How does an object persist for a three-dimensionalist? In contrast with the perdurantist picture, which involves objects persisting in virtue of temporal parts that compose it, Lewis $(1986,202)$ says that three-dimensionalism or endurantism is a theory of persistence that depicts that objects persist by being wholly present at more than one time. However, some have argued that this definition of Lewis made it difficult for us to have a firm grasp of what endurantism really means. For instance, McCall and Lowe have challenged this Lewisian definition of endurance. According to Strorrs McCall and E.J. Lowe, his definition is inapposite since Lewis' usage of "wholly present," and the distinction between perdurantism and endurantism made it difficult for anyone to have a proper understanding of what endurantism means. They $(2009,277)$ argue that "Lewis muddies the waters by giving a confused and ultimately untenable definition of endurance." They $(2009,278)$ started over and provided a "clear and precise" definition of endurance:

An object endures iff (i) it lacks temporal parts, and (ii) it exists at more than one time.

I take it that what McCall and Lowe were referring to when they said that Lewis' distinction of perdurantism and endurantism made it difficult for us to have a proper understanding of endurantism stems from the fact that philosophers have trouble cashing out the term "wholly present."1 Despite this complaint by McCall and Lowe, what remains at the heart of three-dimensionalism is the notion that objects are wholly present at each time it exists. I take it that the definition is given by McCall and Lowe implicitly, or at least it should entail that enduring objects are wholly present. I will leave the cashing out of "wholly present" to the reader, but I think that generally speaking, one cannot do away with this condition if we are talking about enduring objects.

\section{PROBLEMS OF PERSISTENCE}

I mentioned earlier that the reason why terdurantism falls short of its rival theories is that it fails to account for certain problems brought about by persisting objects. These problems are change, cohabitation, and vagueness. I argue that persistence theories should be able to account for these things. Let us explore what constitutes each problem briefly and why they should be accounted for.

It is easy to see why change is a problem when talking about persistence. Ryan Wasserman $(2006,49)$ lists down three conditions that constitute change: 1) Variation (Difference Condition) requires changing objects that have different properties at different times. 2) Opposition (Incompatibility Condition), which states that change requires opposing properties. 3) Identity (Sameness Condition) or the object before the change is the same object after the change. If objects persist, then it is not at all strange for an object to exist at various times while having different, incompatible properties 
at each time it exists. If persistence can be amended such that it satisfies the conditions of change, then persistence theories ought to account for change. An endurantist accounts for change by temporally-relativizing properties (i.e., indexed/ extrinsic). Objects change by having certain properties relative to a time. ${ }^{2}$ Perdurantists account for the problem by saying that objects are composed of temporal parts at different times, and each part has certain properties, simpliciter (i.e., non-indexed/ intrinsic). ${ }^{3}$

Persistence theories must also account for cohabitation or how two (or more) objects of different and incompatible properties can occupy the same space at the same time. This problem arises from the fact that an object can change, but the identity of the resulting object seems to be unidentical to the object before the change hence violating Wasserman's sameness condition. This problem is most commonly illustrated through the statue and the lump thought experiment, where there seem to be two objects present in the resulting object when we mold a lump of clay into a statue. If change ought to be accounted for by persistence theories, and cohabitation occurs under the pretenses of change, then cohabitations ought to be accounted for by persistence theories. An endurantist account for cohabitation is captured by saying that two objects (e.g., statue and clay) are not identical but merely share a relation, "sameness," in that they share all non-modal properties in relation to a time. They are different because their modal properties are different (i.e., the clay may have existed prior to the statue). ${ }^{4}$ The perdurantist would say that coinciding objects are an aggregate of temporal parts. The coincidence of the statue and clay is no stranger than the big road's coincidence with its smaller parts. ${ }^{5}$

Lastly, these theories must account for vagueness. Three things constitute vagueness, according to Rosanna Keefe (1996, 5-6). The first is that objects have borderline cases. Second, objects and predicates seem to lack clear boundaries; this is to say that their boundaries are fuzzy. Lastly, vagueness in objects is said to be susceptible to the sorites paradox. Simply, sorites derives from the Greek word soros, which means "heap." According to the example, when we remove individual pieces one by one from a heap of sand, for instance, we will eventually be left with a solitary grain. The paradox leads to the absurd conclusion that the single grain still counts as a heap. The problem arises whenever we consider the whole lifespan of a persisting object. At what point can we say that the persisting object begins or ceases to exist? Meaning to say, we can pick out two times, $\mathrm{t} 1$ and $\mathrm{t} 2$, and in $\mathrm{t} 1$, the persisting object exists or does not exist, but in $\mathrm{t} 2$, it ceases to exist or does exist, respectively. However, if vagueness is correct, then there must be some time in between $\mathrm{t} 1$ and $\mathrm{t} 2$ where the object is in a fuzzy state, making it difficult to assess whether it is still the same object or not. Endurantists might account for vagueness the same way van Inwagen (1990, 213-227) does. He restricts composition in the sense that composition only occurs if the object composed constitutes life. Inanimate objects like chairs are just simples arranged "chair-wise." Furthermore, the composition is restricted in the sense that there is exactly one suitable set of simples that compose objects. The opposite move is available for perdurantists such that composition is not to be restricted. Lewis (1986, 212-213) argues for an unrestricted composition for a restriction of any sort would be a vague one, and a vague restriction entails that composition is a vague matter which is impossible. This formulation was adopted by Sider (2001, 120-139) and amended it 
as an argument for four-dimensionalism (a term he uses instead of perdurantism) and temporal parts.

\section{TERDURANTISM: NON-PERDURANTIST FOUR-DIMENSIONALISM}

In her paper "Ought a four-dimensionalist to believe in temporal parts?", Kristie Miller (2009, 619-646) formulates a middle-ground position between perdurantism and endurantism. This position serves as an alternative theory of persistence, which allegedly avoids the common arguments against the two. She (2009, 620-621) notes the common problems against endurantism, 1) the problem of cashing out "wholly present" and 2) the unattractive position of relativizing intrinsic properties to times. Against perdurantism, 1a) the perdurantist picture is "ontologically profligate" since a single persisting object is actually a series of shorter-lived objects. 2a) Properties of persisting objects are not their properties but properties of temporal parts. She proposes a non-perdurantist four-dimensionalism picture of persistence or terdurantism (i.e., objects persist by terduring), which allegedly avoids these problems.

It avoids 1a) by denying that objects are not partly present, that there is only one persisting object. It avoids 1) by saying that although terdurantists deny that objects are not partly present, they are not wholly present as well since they are temporally extended. It avoids 2) by denying strict identity at each time. Dropping strict identity over time allows the terdurantist to avoid the problem of adopting temporally-indexed properties. Lastly, it avoids 2 a) by denying that attributing properties to an object could not be "attributing that property to some part of the object," says Miller $(2009,622$ 624 ), since the terdurantist does not have the notion of temporal parts in their picture. Rather, the spacetime worm, since it does not have segments, merely has properties that are instantiated at certain locations similar to Parsons' distributional properties. ${ }^{6}$

What does it mean for terdurantism to be non-perdurantist and fourdimensional, as Miller suggests? A terduring object is four-dimensional because it is extended through time or in Parsons' $(2000,401)$ sense, it satisfies the Dimensionality Thesis which states that "the universe is a four-dimensional manifold of which one of the dimensions is time." Furthermore, they are also consistent with the perdurantist notion that objects are spacetime worms. However, these objects are non-perduring since these worms are not segmented, meaning they lack temporal parts. The "nonperdurantist" part involves a denial of the so-called Rich Perdurantist Thesis. ${ }^{7}$ Simply put, this thesis argues that temporal parts exist when it comes to persisting objects. Terdurantism, then, denies that persisting objects have temporal parts. Miller's motivation for terdurantism can be simplified as follows:

P1: perdurantism and endurantism each faces some serious problems

$\mathrm{P} 2$ : if a theory of persistence avoids these problems, then it is a viable theory of persistence

P3: terdurantism avoids these problems

C: Terdurantism is a viable theory of persistence (modus ponens from P2 to P3) 
Miller $(2009,626)$ begins her definition of terdurantism with terduring simples. The terdurantist picture of persistence is committed to the existence of terduring simples" ${ }^{8}$, which are "four-dimensional partless objects that are not strictly identical across time." These simples fuse to compose terduring composite objects or fusions. Miller (2009, 631) stipulates:

A complex object $\mathrm{O}$, terdures iff: (i) $\mathrm{O}$ persist through some temporal interval which contains temporal instants $t$ and $\mathrm{f}^{*}$ and (ii) the threedimensional slice of $\mathrm{O}$ at $\mathrm{t}$ is not strictly identical to the three-dimensional slice of $\mathrm{O}$ at $\mathrm{t}^{*}$ and (iii) it is not the case that for every instant $\mathrm{t}$ in and subinterval ? in $\mathrm{T}$, that there is some object $\mathrm{O}^{*}$ that exists at exactly that instant or sub-interval, and which overlaps every part of $\mathrm{O}$ at that instant or during that sub-interval.

The first premise implies that the object persists. The second implies that the object does not persist by enduring since the object is extended through time, and it denies that the object is strictly identical at each time it exists. The third condition implies that terduring objects are not segmented into maximal temporal parts so it does not persist by perduring. Furthermore, the third condition entails that all conditions of the rich perdurantist thesis are false. This is, for Miller, the stronger sense of terdurantism.

These fusions and the simples which compose them are "ontologically basic" and are responsible for the existence of ordinary, everyday objects which are regarded as non-fusions. This picture tells us that worldly objects (i.e., non-fusions) owe their existence to fusions. They are related to one another through a relation called compilation. Miller $(2009,634)$ defines the relation as:

Compilation: A fusion $\mathrm{F}$ compiles a non-fusion $\mathrm{O}$ at a time $\mathrm{t}$ iff (i) $\mathrm{F}$ and $\mathrm{O}$ exist at $\mathrm{t}$, and (ii) The existence of $\mathrm{O}$ at $\mathrm{t}$ is entailed by $\mathrm{F}$ existing at $t$ and $F$ having the intrinsic properties at $t$ that it does and (iii) there is no proper part of $\mathrm{F}$ whose existence entails the existence of $\mathrm{O}$ at $\mathrm{t}$.

Miller (2009, 635) summarizes the terdurantist ontology in the following manner: 1) there are fusions of terduring simples which form composite objects called terduring fusions. 2) Fusions are related to their parts in a temporally-unmodified manner. 3) Ordinary objects are not related to their parts in this manner; they are also not fusions of parts at times. 4) Ordinary objects, then, are non-fusions that are related to terduring fusions at different times through compilation. 5) Parts of non-fusions are parts they inherit from the terduring fusion, which compiles them at that time

\section{ARGUMENT FROM PROBLEM-SOLVING}

Given the three problems, I claim that any theory of persistence should be able to account for these things. Doing so bolsters any given theory in terms of explanatory power and feasibility because it provides an avenue for its defenders to test and apply 
the core concepts of their respective theories. This is true in the case of perdurantism and endurantism, wherein philosophers who support one or the other theory are able to motivate their positions further on the grounds that they can account for these problems. The fact that there is no consensus as to which among the two gives us the correct picture of persistence should not worry us. What we should take away from the long-standing dispute and the vast literature that comes along with it is that philosophers have, over the years, been able to argue for their sides by applying them in certain issues where persistence matters. My argument against terdurantism and for the necessity of accounting for the persistence problems can be formalized as follows:

P1: Any plausible theory of persistence should account for the problems of persistence: change, cohabitation, vagueness

P2: If terdurantism is a plausible theory of persistence, then it should be able to account for these problems

P3: Terdurantism fails to account for any of the three

C: Therefore, terdurantism is not a plausible theory of persistence

The key premise here is $\mathrm{P} 1$, as it allows us to evaluate and judge any theory of persistence based on how they account for the persistence problems. Any theory which fails to do so should be rejected, or at the least, be seen as unattractive compared to those that can. This means that if the theory can only account for one or two of the problems or none at all, then one should question whether or not it is a good theory of persistence, to begin with, if not reject it altogether. $\mathrm{P} 2$ is a deduction from $\mathrm{P} 1$. The conclusion is achieved via modus tollens from P2 to P3 (where P3 is a rejection of P2's consequent).

\section{POSSIBLE OBJECTIONS AND REPLIES}

PO1: Why should we favor one theory over the other if all of them have their fair share of criticisms? In other words, why prefer endurantism and perdurantism over terdurantism if the former two have been criticized by philosophers from opposing sides?

Reply: Although philosophers from opposing sides challenge each other's accounts to the problems by claiming that the other's theory is problematic or that "it fails to hold water," philosophers from their own respective sides can effectively respond to each criticism since their theories already have a strong foundation. On the side of perdurantism, we have temporal parts, while on the side of endurantism, we have the notion of strict identity over time and wholly-present persisting objects. Philosophers of persistence have, over the years, developed their respective theories based on these core concepts. Therefore, we have reason to prefer perdurantism or endurantism over terdurantism despite the criticisms of opposing parties. This is due to the substantial work done by philosophers to develop and defend their theories. Furthermore, it is through constant criticisms where philosophers are able to identify the weaknesses of their theories. With this, they can take the necessary steps to further 
improve them. Terdurantism is a relatively young theory of persistence, and there is very little written about it in the literature. Although Miller did criticize the theory at hand, the lack of responses in defense of terdurantism makes the other two theories much more preferable.

PO2.1: What about theories that do not yet answer all three problems? Should they be rejected outright just because their theory is relatively younger? One might say that P1 is too strong since it immediately rules out new, possible contenders should they fail to solve the persistence problems. Given an ample amount of time and work to advance the new theory, it could potentially give a tenable account of the three problems and thus rival our older theories.

Reply: P1 merely serves as a criterion for us to check whether or not one's theory of persistence is any good or not when it comes to its explanatory power and the flexibility of its core ideas when it comes to the three problems. Should it fail to give a tenable account of the persistence problems, then maybe it is because not much work has been done to advance the theory or that the core ideas of the theory are problematic in themselves. We need not reject them outright, and any work to advance the theory is welcome. But as long as it fails to give an account of the three problems, we have little reason to prefer it over older, more mature theories.

PO2.2: What about younger theories that can give a fairly tenable account to only one or two of the problems? P1 is too strong since it would rule out theories that can account for one or two of the problems. However, with some development, it could potentially account for all three.

Reply: My reply to this objection is similar to my reply to PO2.1. Again, we need not reject them outright, and given substantial work, the theory in question might provide a viable account for all three problems. However, we should, at the least, see the theory in question as unattractive. As it stands, we have no reason to prefer such a theory over its much older counterparts. Given that the solutions which the theory provides are tenable, it would be insufficient since all the problems need to be accounted for when talking about persistence. Just failing to take into account just one of the three should already serve as a red flag that the theory may not be a viable one when it comes to explaining an object's persistence. One may want to reconsider if the theory in question is worth salvaging at all.

\section{REJECTING A TERDURANTIST ACCOUNT OF THE PROBLEMS}

The remaining portion of the paper is dedicated to showing how terdurantists might account for the problems. Following each account will be a response showing how the terdurantist solutions fail to solve the problem

\section{On Change}

To account for change, the perdurantist notion of property instantiation through temporal parts that have these properties simpliciter is not available for the terdurantist since they would deny that temporal parts exist. The terdurantist also denies that objects are not wholly present at each time they exist. So the endurantist move of 
temporally relativizing properties and maintaining that there is strict identity overtime when it comes to persisting objects is also unavailable. What the terdurantist could do is import Miller's notion of irreducibly regionalized instantiated properties. Talk of property instantiation is talk of suitable candidates which have these properties simpliciter. For the perdurantist, temporal parts are said to instantiate properties in this manner. For the endurantist, since they hold that persisting objects are wholly present at each time they exist, they have to appeal to instantiating properties at times. Since neither is available for the terdurantist, an amendment of the endurantist position can be done such that instead of relativizing properties to times, they could appeal to relativizing properties to spacetime regions "where these regions need not correspond to some three-dimensional 'slice' of the four-dimensional worm." According to Miller (2009, 640). Properties of terduring fusions and the simples that compose them are not properties they have simpliciter but properties they instantiate in relation to regions with these properties.

Moreover, since both these objects do not have parts which can be considered viable candidates for instantiating these properties simpliciter, Miller $(2009,640)$ says, "the terdurantist must appeal to some irreducible instantiation relation: at-R (at a region) or R-ly (in a regional manner)." This suggests that since ordinary objects (nonfusions) owe their existence to the fusions which compile them, they also adopt the properties had by those fusions. Therefore, in the terdurantist picture of change, when talking about different properties, a non-fusion's property at each time it exists can be cashed out in terms of the properties instantiated by fusions which compile them at regions. Regions that have the properties simpliciter.

In order to refute this account, we must first go back to Miller's identified key objections 2) and 2a) against endurantism and perdurantism, respectively, in that they both depict an unsatisfactory picture of the nature of intrinsic properties. She says that it can be argued against both positions that the properties of a persisting object are not properties of the object itself. For the perdurantist, temporary properties are properties had by temporal parts, simpliciter. For the endurantist, temporary properties are instantiated in a temporally-modified manner. The terdurantist position is an amendment of the endurantist position with the exception of regionally-modified properties instead of temporal ones. Since the endurantist position is problematized on the basis of 2), then a terdurantist account of change can be challenged in a similar manner. That the properties had by non-fusions are not really properties they have, simpliciter but properties that are ultimately reducible to regions. For this reason, Miller $(2009,641)$ fails to see how terdurantism fares any better than endurantism.

\section{On Vagueness}

Recall that the terdurantist picture of persistence involves the existence of ontologically basic objects: terduring simples and fusions composed of simples. Ordinary objects or non-fusions are intimately linked with the fusions through a compilation relation. Vagueness can be accounted for by the terdurantist the same way endurantists do: by restricting composition. According to Miller, terduring simples are typically scattered and do not compose anything. It is only contingently that they fuse 
to form a composite fusion, which, as a result, compiles a non-fusion. She $(2009,637-$ 638) says, "it is when these simples thus coalesce, that we are inclined to say that their fusion compiles, at a time, some non-fusion." Given this, the terdurantist could say that there really is no vagueness because what is required for a case of composition to occur are all necessary simples that form the fusion. Omitting or adding a simple from and to the fusion, respectively, will either result in a completely different composite object or failure to compose anything at all; it is only during those instances where fusions occur that non-fusions exist.

The most straightforward objection would come from David Lewis. He (1986, 211) argues that when it comes to composition, there is no vagueness, and any class of objects can compose something. He $(1986,212)$ further claims that vagueness is a "semantic indecision" and exists only within thought and language. There seems to be vagueness because no one has taken it upon themselves to define and make explicit the boundaries of terms such as bald, tall, heap, etc. Rather than restrict composition, Lewis $(1986,212)$ argues that no matter how scattered and unrelated objects may be, they always compose something. This is because any sort of restriction on composition would require a vague one, which leads to the impossible conclusion that whether composition occurs or not is also a vague matter. Either composition occurs, or it does not.

Another objection raised by Miller has to do with the terdurantist commitment to the existence of terduring simples, fusions, and non-fusions. Recall that objection 1a) charges perdurantism with an "ontologically profligate" picture. In order to explain persistence, the perdurantist must appeal to several temporal parts that make up the whole four-dimensional segmented worm. The terdurantist holds that the basic objects are fusions to which non-fusions owe their existence to. Since non-fusions exist at various times, then there must be enough fusions to compose all non-fusions at each time it exists. One can already imagine how abundant the number of fusions required must be to compile a single non-fusion at times which it exists. If such is the case, Miller $(2009,639)$ argues, "terdurantism is quite ontologically profligate" and fares no better than perdurantism with respect to objection 1a).

\section{On Cohabitation}

What the terdurantist might do to account for cohabitation is explain it in terms of the compilation relation which holds between fusions and the non-fusions they compile. Since the latter is nothing over and above the former, which compiles it, properties had by fusions are inherited by non-fusions. The notion of instantiated regionalized properties is also crucial. Perdurantists hold that cohabitation is not a strange occurrence at all, no stranger than the coincidence of a long road with its shorter parts. In contrast, the terdurantist holds that since all properties are regionally spread out and merely instantiated by fusions at times, then we can liken the entire spacetime region to a long road with various parts. This picture is consistent with terdurantism since it is the region, not the fusions, which has parts. Whenever we consider a clay molded into a statue, we can simply say that the fusions responsible for compiling the clay instantiated clay-like properties from a part of a region. Such too is 
the case for the statue. The fusions which compile the statue at the time it was formed instantiated clay and statue-like properties from the region which has those properties. I decided to discuss the cohabitation account last because the objections against it are similar to those before. While coherent, the account still requires us to appeal to an instantiation relation wherein the properties of non-fusions be traced down to fusions then to regions. It was already established from the argument against change that such an account is problematic because properties of objects are not really properties that they have simpliciter. In this respect, the picture still falls short of 2) and 2a). We can also imagine how much fusions must be conceived just to capture the process of molding a lump into a statue. At each stage and time, there must be quite a number of fusions required which compile the lump, the statue, and the "in-between" objects. The account falls short of objection 1a).

\section{CONCLUSION}

For any theory of persistence, it is not enough that the theory in question explains how an object persists. Persistence is accompanied by three further problems: change, cohabitation, and vagueness. Change stems from the fact that objects can persist and have different, incompatible properties at various times. Cohabitation stems from the fact that a persisting object can change such that the identity of the resulting object seems to be that of two unidentical objects occupying the same space at the same time. Vagueness stems from the fact that an object can go in and out of existence, yet it is not clear at which point during the process do we draw the line between existence and non-existence. Any theory of persistence ought to give us a complete and coherent picture of persistence. The picture will be incomplete if the theory fails to account for even one of these three problems. Any theory which fails to account for any of the problems should be seen as unsatisfactory, if not rejected altogether. Since terdurantism fails to do just this, then it should be seen as an unsatisfactory theory of persistence. More importantly, we now have at our disposal a device by which we can assess and test any theory of persistence (old and new alike) based on their capability to account for the persistence problems.

\section{NOTES}

1. For different interpretations of "wholly present," see Theodore Sider (1997, 11-16).

2. See McCall \& Lowe (2009) for a more in-depth defense of the endurantist account.

3. See Sider (1997) \& (2008) for a more in-depth account of the perdurantist position.

4. See Moyer (2006) for an articulation of the problem and the endurantist response.

5. See Sider (1997) \& (2008). 
6. In order to make temporally-indexed properties intrinsic, Parsons (2000) introduces the notion of distributional properties. These are properties that an object has intrinsically but can be used by endurantists to explain change and escape the problem of temporary intrinsic. They are also disjunctive. Simply, distributional properties are properties that are instantiated by enduring objects at different times. An example of a distributional property is the heat at which metals expand. If we consider two different metals and utter "expanded-at-t1," then we would either refer to metal one or metal two. Either way, the heat at which each metal expands is intrinsic to them

7. Rich Perdurantist Thesis (RPT), for Miller $(2009,627)$, is the theory that: for any persisting object $\mathrm{O}$ that exists during interval $\mathrm{T}$, and for every instant $\mathrm{t}$ in $\mathrm{T}$, and every sub-interval I in T (i), there is some object $\mathrm{O} 1$ that exists at and only at $\mathrm{t}$, (ii) there is some object $\mathrm{O} 2$ that exists during and only during I, (iii) $\mathrm{O} 1$ overlaps every part of $\mathrm{O}$ at $\mathrm{t}$, (iv) $\mathrm{O} 2$ overlaps every part of $\mathrm{O}$ during I and (v) $\mathrm{O} 1$ and $\mathrm{O} 2$ are proper parts of $\mathrm{O}$.

8. According to Miller $(2009,626)$, A simple $S$ terdures iff: (i) $S$ is mereologically simple, and (ii) $\mathrm{S}$ persists through some temporal interval which contains instants $t$ and $t^{*}$ and (iii) the three-dimensional slice of $S$ at $t$ is not strictly identical to the three-dimensional slice of $\mathrm{S}$ at $\mathrm{f} *$.

\section{REFERENCES}

Keefe, Rosanna. 1996. Vagueness: A reader. Edited by Rosana Keefe \& Peter Smith. Cambridge: MIT Press.

Lewis, David. 1986. On the plurality of worlds. Oxford: Blackwell.

McCall, Storrs \& Lowe, E. J. 2009. The definition of endurance. Analysis, 69(2), 277280.

Miller, Kristie. 2009. Ought a four-dimensionalist to believe in temporal Parts? Canadian Journal of Philosophy, 39(4), 619-646.

Moyer, Mark. 2006. Statues and lumps: A strange coincidence? Synthese, 148(2), 401-423.

Parsons, Josh. 2000. Must a four-dimensionalist believe in temporal parts? The Monist, vol. 83, no. 3, pp. 399-418.

Sider, Theodore. 1997. Four-dimensionalism. Philosophical Review, 197-231.

Sider, Theodore. 2001. Four-dimensionalism: An ontology of persistence and time. Oxford University Press.

Sider, Theodore. 2008. Three-dimensionalism versus four-dimensionalism. In Contemporary debates in metaphysics. Edited by Theodore Sider, John Hawthorne, Dean W. Zimmerman. Oxford: Blackwell.

Van Inwagen, Peter. 1990. Material beings. New York: Cornell University Press.

Wasserman, Ryan. 2006. The problem of change. Philosophy Compass, 1(1), 48-57. 